\title{
Functionalized Akiyama tips for magnetic force microscopy measurements
}

\author{
Markus Stiller, ${ }^{1, *}$ José Barzola-Quiquia, ${ }^{1}$ Pablo D. Esquinazi, ${ }^{1}$ Soraya \\ Sangiao, ${ }^{2,3}$ José M. De Teresa, ${ }^{4,2,3}$ Jan Meijer, ${ }^{5}$ and Bernd $\mathrm{Abel}^{6}$ \\ ${ }^{1}$ Division of Superconductivity and Magnetism, Felix-Bloch Institute \\ for Solid-state Physics, University of Leipzig, 04103 Leipzig, Germany \\ ${ }^{2}$ Laboratorio de Microscopías Avanzadas (LMA), Instituto de Nanociencia de Aragón (INA), \\ Universidad de Zaragoza, E-50018 Zaragoza, Spain \\ ${ }^{3}$ Departamento de Física de la Materia Condensada, \\ Universidad de Zaragoza, E-50009 Zaragoza, Spain \\ ${ }^{4}$ Instituto de Ciencia de Materiales de Aragón, CSIC-Universidad de Zaragoza, 50009 Zaragoza, Spain \\ ${ }^{5}$ Division of Nuclear Solid State Physics, Felix-Bloch Institute for \\ Solid-state Physics, University of Leipzig, 04103 Leipzig, Germany \\ ${ }^{6}$ Leibniz Institute of Surface Modification, 04318 Leipzig, Germany
}

(Dated: October 4, 2017)

\begin{abstract}
In this work we have used focused electron beam induced deposition of cobalt to functionalize atomic force microscopy Akiyama tips for application in magnetic force microscopy. The grown tips have a content of $\approx 90 \%$ Co after exposure to ambient air. The magnetic tips were characterized using energy dispersive X-ray spectroscopy and scanning electron microscopy. In order to investigate the magnetic properties, current loops were prepared by electron beam lithography. Measurements at room temperature as well as $4.2 \mathrm{~K}$ were carried out and the coercive field of $\approx 6.8 \mathrm{mT}$ of the Co tip was estimated by applying several external fields in the opposite direction of the tip magnetization. Magnetic Akiyama tips open new possibilities for wide-range temperature magnetic force microscopy measurements.

PACS numbers: 68.37.Rt,07.79.Pk,81.15.Jj
\end{abstract}

\section{INTRODUCTION}

Magnetic force microscopy (MFM) is an essential tool to probe the local stray fields of materials and can access information from nanometer to micrometer length scales. This is important for experimental work in the area of nanotechnology, where MFM offers superior spatial resolution compared to superconducting quantum interference measurements [1]. Thus, MFM has been used to investigate and to manipulate vortices in superconductors [2-4] or ferromagnetic domain walls in thin films [5-7] and micro/nano-structures [8]. However, most MFM systems are restricted to room-temperature measurements, lacking the possibility to explore the temperature dependence of magnetic and/or superconducting properties in such samples. Another crucial factor is, that conventionally AFM devices are operated using a laser to detect the cantilever oscillation, which implies a working place of the order of tens of centimeters, while for AFM using Akiyama tips, the working place is reduced to a few centimeters, making it possible to place them inside a cryostat or employ multi-probe scans. Furthermore, these tips can be used in darkness and thus allow for measurements inside tubes or a combination of AFM/MFM with scanning electron, optical or confocal microscopy. An example of Akiyama tips used for magnetic measurements is the combination with diamonds containing NVcenters which are glued at the end of the tip [9]. In addi-

\footnotetext{
*markus@mstiller.org
}

tion, low-temperature measurements can be useful to rule out topographic or electrostatic contributions [10]. Often, samples undergo a variety of phase transitions upon cooling, such as magnetic quantum tunneling or magnetic anisotropies of small particles that exhibit superparamagnetism at room temperature. The hardware for selfoscillation and self-sensing tips requires less space and can be placed easily in a cryostat for temperature dependent measurements. Low-temperature MFM has been used to study micrometric aggregates of a paramagnetic gadolinium acetate complex [11] or magnetic switching of ferromagnetic Prussian blue analogue nanoparticles [12] or single molecule magnets [10].

Moreover, the MFM device itself profits from low temperature, since thermally induced drifts and piezo creeps are smaller, which is very useful for devices where closedloop scanning is not possible. The cryo-cooled AFM device used for this work, was operated using Akiyama tips, for which no magnetic tips are commercially available. In order to produce magnetic tips, focused electron beam induced deposition (FEBID) of Co was used. Current loops were prepared to characterize the tips and also for comparison using standard MFM tips with a standard AFM/MFM device. Using detailed theory and computing the equations with standard programs, simulations were done to compare with the results.

\section{EXPERIMENTAL PROCEDURE}

For the experiments commercial Akiyama tips (Nanosensors) designed for AFM were used, with a spring 

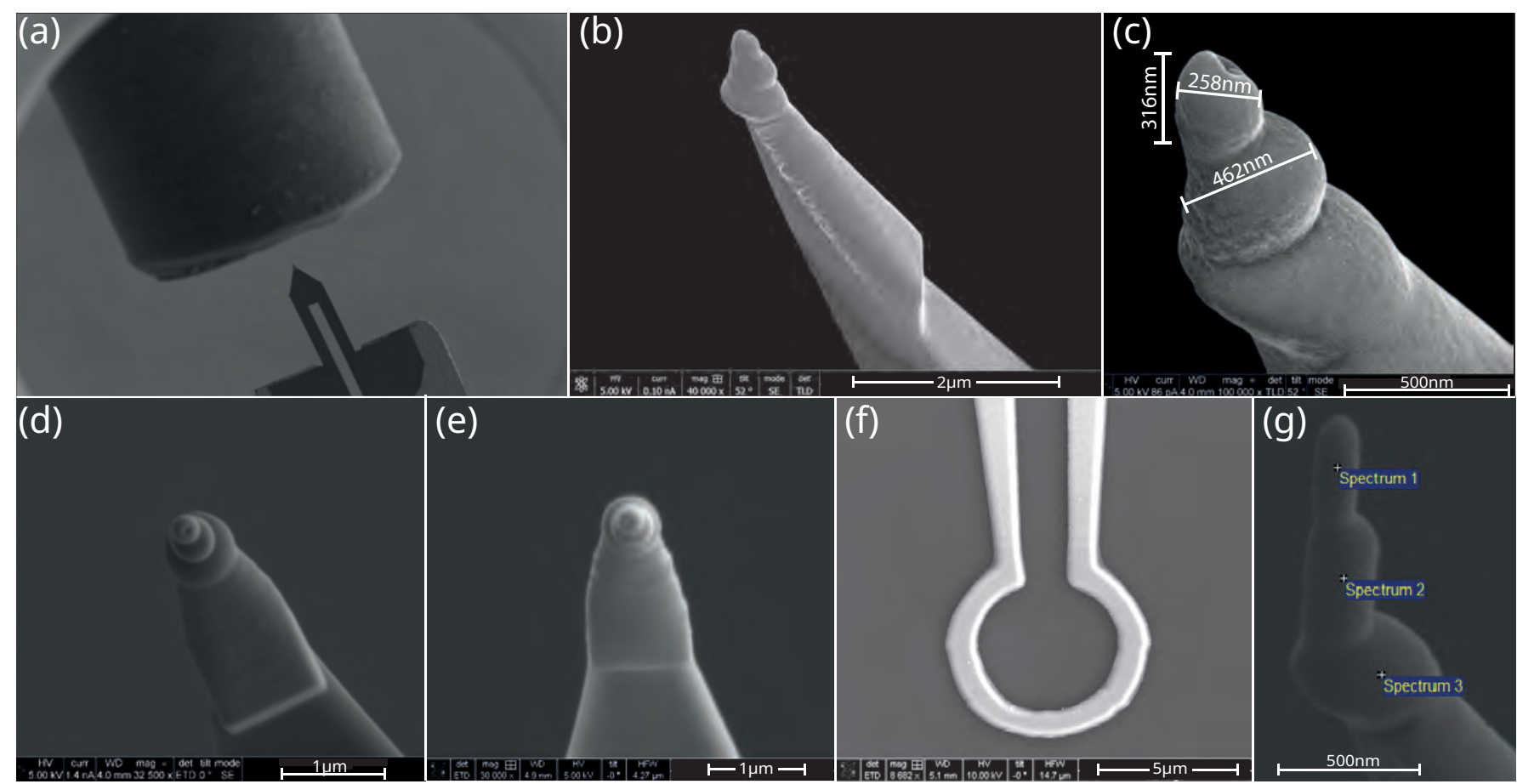

Fig. 1. The Akiyama cantilever and gas injection system is shown in (a). A deposited magnetic tip can be seen in (b) and (c). Pictures (d) and (e) show the magnetic tip before (d) and after measurements (e). The current loop to investigate the response on a magnetic field can be seen in (f), and the crosses in (g) indicate where the EDX spectra were recorded.

constant of $k=5 \mathrm{~N} / \mathrm{m}$. For comparison, a conventional MFM device (Veeco) with standard MFM tips (Bruker, $k=3 \mathrm{~N} / \mathrm{m}, \mathrm{Q}=220, \mathrm{r}=35 \mathrm{~nm})$ was used. The measurements with Akiyama tips were done using a AFM/CFM device (Attocube) placed in a He-cooled cryostat (Oxford), low-temperature measurements had to be performed using a phase-locked loop due to the large quality factor $Q=2757$ at $T=4.2 \mathrm{~K}$. Magnetic Akiyama tips have been prepared by FEBID using a dual-beam Helios 600 system (FEI) that integrates an electron beam and an ion column, see Figs. 1((a)-(e)). FEBID is an in-situ deposition method, allowing the production of nano-tomicrometer-sized structures with desired thicknesses and shape.

During the deposition, the focused electron beam current was set to $0.17 \mathrm{nA}$ at $5 \mathrm{kV}$ acceleration voltage and a $\mathrm{Co}_{2}(\mathrm{CO})_{8}$ gas precursor was injected. Precursor molecules, delivered onto the substrate surface by means of a nearby gas-injection system, are dissociated by the electron beam irradiation, giving rise to a deposit with the same shape of the beam scanning [13]. FEBID allows the precise growth of a magnetic tip at the apex of a cantilever with controlled tip dimensions $[14,15]$. In the present case, a continuous electron irradiation on a single spot has been used for the growth of the magnetic tip at the apex of the cantilever, see Figs. 1((b)-(e)). Energydispersive X-ray (EDX) spectra were acquired after exposing the cantilevers to ambient conditions for several minutes, which gives rise to a saturated surface oxidation layer of $\approx 5 \mathrm{~nm}[16]$, see Fig. $1(\mathrm{~g})$.
The tips were grown as follows: first a square-like structure was deposited as basis for the tip (rectangle, $(2 \times 1 \times 0.1) \mu \mathrm{m}$, deposition time $t=252 \mathrm{~s})$, followed by two circles, with radii $300 \mathrm{~nm}(t=19 \mathrm{~s})$ and $100 \mathrm{~nm}$ $(t=11 \mathrm{~s})$. The resulting tip size was $\approx 250 \mathrm{~nm}$, see Figs. 1((a)-(d)), due to the broadening effect characteristic of the growth by FEBID [17]. In Fig. 1(e) the tip after measurement can be inspected, and compared with Fig. 1(d), it is obvious that deposited material is robust and suitable for AFM/MFM measurements.

The current loops were patterned with electron beam lithography, and $\mathrm{Cr} / \mathrm{Au}$ with a thickness of $\approx 7 \mathrm{~nm} / \approx 200 \mathrm{~nm}$, was sputtered, see Fig. 1(f). The loops used at room temperature have a radius of $4 \mu \mathrm{m}$ and $\approx 150 \mathrm{~nm}$ width, whereas the current loops used at low temperatures have a radius of $2.4 \mu \mathrm{m}$ and a width of $\approx 1 \mu \mathrm{m}$.

\section{RESULTS AND DISCUSSION}

Using EDX, the Co purity was determined to be between 85 and 92 at \%., see Table I. For such Co content, electron holography measurements in Co nanowires indicate that the magnetization along the long wire axis is around $1 \mathrm{~T}[16]$. The $\mathrm{C}$ content given by EDX are all the same within the experimental error, which is always larger than 2-3\%; i.e. that the values are comparable. Further, studies of Co nanostructures indicate that the structures are composed of a rich Co core and an (stable) 


\begin{tabular}{llll}
\hline \hline Spectrum & $\mathrm{C}(\mathrm{At} \%)$ & $\mathrm{O}(\mathrm{At} \%)$ & Co $(\mathrm{At} \%)$ \\
\hline 1 & 6.88 & 1.31 & 91.81 \\
2 & 7.42 & 0.83 & 91.75 \\
3 & 12.23 & 1.54 & 86.23 \\
mean & 8.84 & 0.83 & 89.93 \\
\hline \hline
\end{tabular}

TABLE I. EDX results of a magnetic Akiyama tip at positions shown in Fig. 1(g). The spectra were recorded after exposure to ambient air.

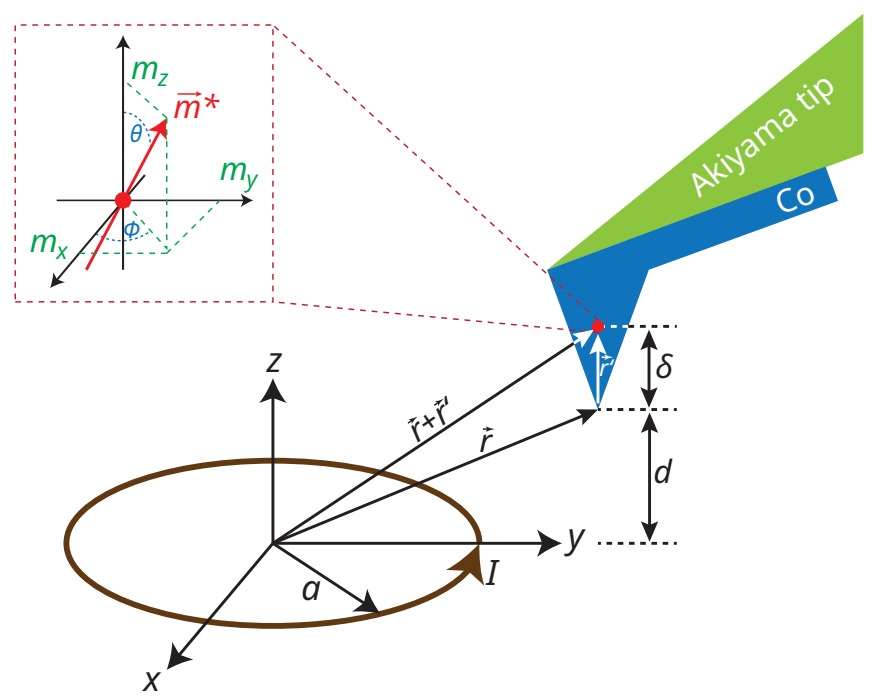

Fig. 2. Geometry of current loop and Akiyama tip with deposited Co. The effective magnetic moment $m^{*}$ of the tip is treated as dipole with arbitrary angle.

oxidized cobalt surface, which can be a reason for different values at positions with different surface/volume ratios [18].

In order to quantify the results, current loops were used to generate magnetic fields. Considering a current loop of radius $a$, see Figs. 1 (f) and 2, located in the $x-y$ plane, centered at origin and carrying a current $I$, the generated magnetic field in Cartesian coordinates is [19]:

$$
\begin{aligned}
B_{x} & =\frac{C \cdot x \cdot z}{2 \alpha^{2} \beta \rho^{2}}\left[\left(a^{2}+r^{2}\right) E\left(k^{2}\right)-\alpha^{2} K\left(k^{2}\right)\right], \\
B_{y} & =\frac{C \cdot y \cdot z}{2 \alpha^{2} \beta \rho^{2}}\left[\left(a^{2}+r^{2}\right) E\left(k^{2}\right)-\alpha^{2} K\left(k^{2}\right)\right] \\
& =\frac{y}{x} B_{x} \\
B_{z} & =\frac{C}{2 \alpha^{2} \beta}\left[\left(a^{2}-r^{2}\right) E\left(k^{2}\right)+\alpha^{2} K\left(k^{2}\right)\right],
\end{aligned}
$$

where $\rho^{2}=x^{2}+y^{2}, r^{2}=x^{2}+y^{2}+z^{2}, \alpha^{2}=a^{2}+r^{2}-$ $2 a \rho, \beta^{2}=a^{2}+r^{2}+2 a \rho, k^{2}=1-\alpha^{2} / \beta^{2}, C=\mu_{0} I / \pi$, $K$ and $E$ are the elliptic integrals of first and second kind, respectively. The magnetic force derivative for an arbitrary cantilever orientation is given by [20]

$$
\begin{aligned}
F^{\prime}(\vec{r}) & =\int_{\operatorname{tip}} \sum_{i=x, y, z} \sum_{j=x, y, z} \sum_{k=x, y, z} n_{j} n_{k} M_{i}^{T}\left(\vec{r}^{\prime}\right) \\
& \times \frac{\partial^{2} B_{i}\left(\vec{r}+\vec{r}^{\prime}\right)}{\partial r_{j} \partial r_{k}} d V^{\prime}
\end{aligned}
$$

where $\vec{M}^{T}$ denotes the tip magnetization. Assuming that the sample magnetization and $\vec{M}^{T}$ are independent of each other, that the cantilever is parallel to the sample surface, i.e. $\hat{n}=\hat{z}$, and that tip is a point dipole, one finds that

$$
F^{\prime}(\vec{r})=m_{x} \frac{\partial^{2} B_{x}}{\partial_{z}^{2}}+m_{y} \frac{\partial^{2} B_{y}}{\partial_{z}^{2}}+m_{z} \frac{\partial^{2} B_{z}}{\partial_{z}^{2}},
$$

with

$$
\vec{m}^{*}=\left(\begin{array}{c}
m_{x} \\
m_{y} \\
m_{z}
\end{array}\right)
$$

where $m^{*}$ is the effective magnetic moment of the deposited tip. This provides the possibility to include an arbitrary angle of the tip point dipole into the simulations, see Fig. 2; Matlab $b^{\mathrm{TM}}$ was used to calculate the derivatives of Eqs. (1). The phase shift of the cantilever can then be simulated with

$$
\Delta \varphi=-\frac{Q}{k}\left(\frac{\partial F}{\partial z}\right)=\frac{2 Q}{\omega_{0}} \Delta \omega,
$$

where $Q$ is the quality factor, $k$ the spring constant, $\omega_{0}$ and $\Delta \omega$ are the resonance frequency and the frequency shift, respectively. Assuming that $\vec{M}^{T}$ is perfectly aligned parallel to $\hat{z}$, i.e. $m_{y}=m_{x}=0$, then the phase shift along the $z$-axis of the loop is given as:

$$
\Delta \varphi_{z}=\frac{3 \mu_{0} a^{2} \operatorname{Im}_{z} Q}{2 k}\left[\frac{a^{2}-4(d+\delta)^{2}}{\left(a^{2}+(d+\delta)^{2}\right)^{7 / 2}}\right],
$$

with $z=d+\delta$, where $d$ is the scan height and $\delta$ is the distance between the Co tip and the center of the magnetic dipole. This phase shift (Eq. (5)) is the difference of the phase between the center of the ring and the phase signal at a large enough distance away from the current ring with no stray fields present (as indicated in Fig. 4(e)). This method has the advantage that the magnetization will not be changed when scanning with the magnetic tip or when applying an external magnetic field, which could be the case when using a ferromagnetic sample. Also a linear current wire could be used. However, this is not accurate because the MFM image would have to be analyzed above an edge of the wire, in order to get the $z$-component of the tip stray fields [21]. This not only implies possible topographic influence but also electrostatic distortion might occur due to contact potential between tip and the current carrying wire [21], which was seen clearly in the measurements using the Akiyama 

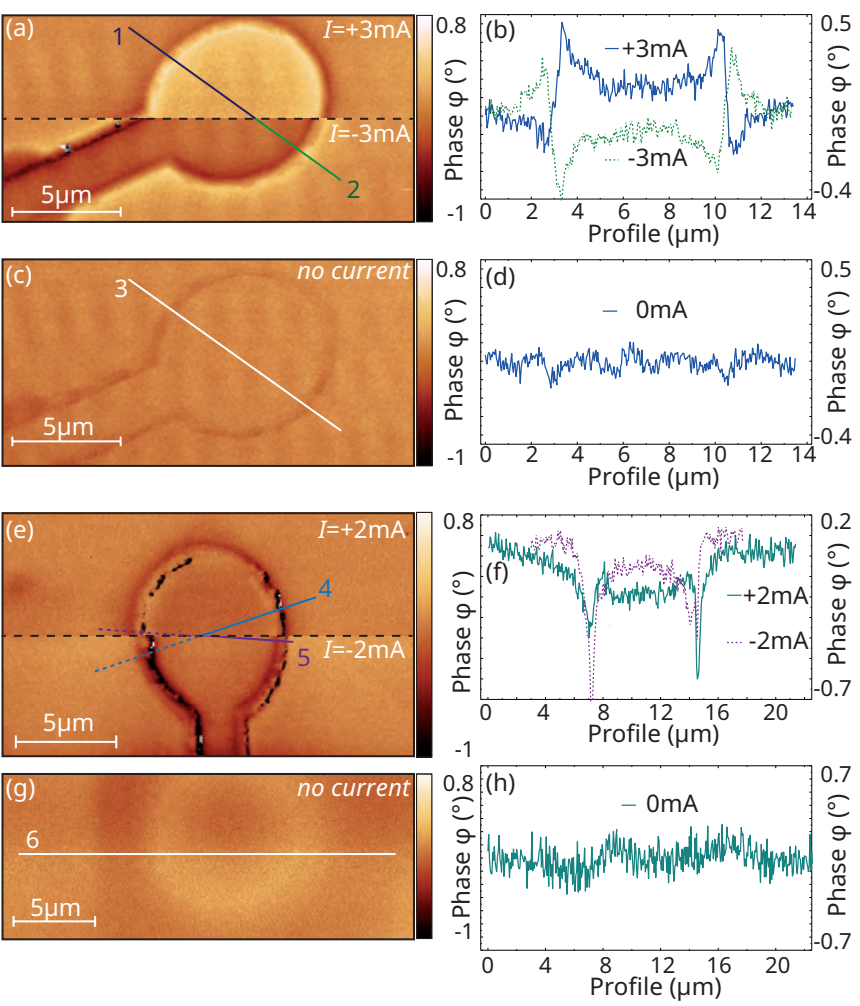

Fig. 3. Magnetic force microscopy images at room temperature of a current loop using a standard MFM tip and standard device for applied currents of $I= \pm 3 \mathrm{~mA}$ (a) and no applied current(c). Line scans as indicated can be seen in ((b)\&(d)). The results using an Akiyama tip with applied currents of $I= \pm 2 \mathrm{~mA}$ are shown in (e), with no current applied in (g). The corresponding line scans can be found in $((\mathrm{f}) \&(\mathrm{~h}))$.

tip. This could be avoided when covering the wire with an insulating film and a gold film on top, which then can be connected to the tip in order to electrostatically shield the tip [22].

In Fig. 3 the results of MFM measurements at room temperature are shown. The commercial tip together with the standard AFM/MFM device yield the expected results, see Fig. 3((a)-(d)). In Fig. 3(a) the top MFM image shows the results when a current of $I=+3 \mathrm{~mA}$ is applied, the bottom image was measured with a current of $I=-3 \mathrm{~mA}$. The magnetic field at the center of the loop was $B_{c} \approx \pm 471 \mu \mathrm{T}$. The scan height of $90 \mathrm{~nm}$ has only a low influence on the field strength, as the 1/e magnetic decay length for a current loop is roughly equal to the radius [23]. The two lines indicate the spectra shown in Fig. 3(b), i.e. spectrum 1 for positive and spectrum 2 for a negative applied current, respectively. The phase shift $\Delta \varphi_{z}$ was found to be $\pm 0.15^{\circ}$. In Fig. 3(c) and (d) the results for zero current are shown. Besides small topographic effects when scanning across the wire, no signal is found at the center of the loop.

The results for Akiyama tip at room temperature can be seen in Fig. 3((e)-(h)), with applied currents of $I= \pm 2 \mathrm{~mA}$ resulting in magnetic fields of
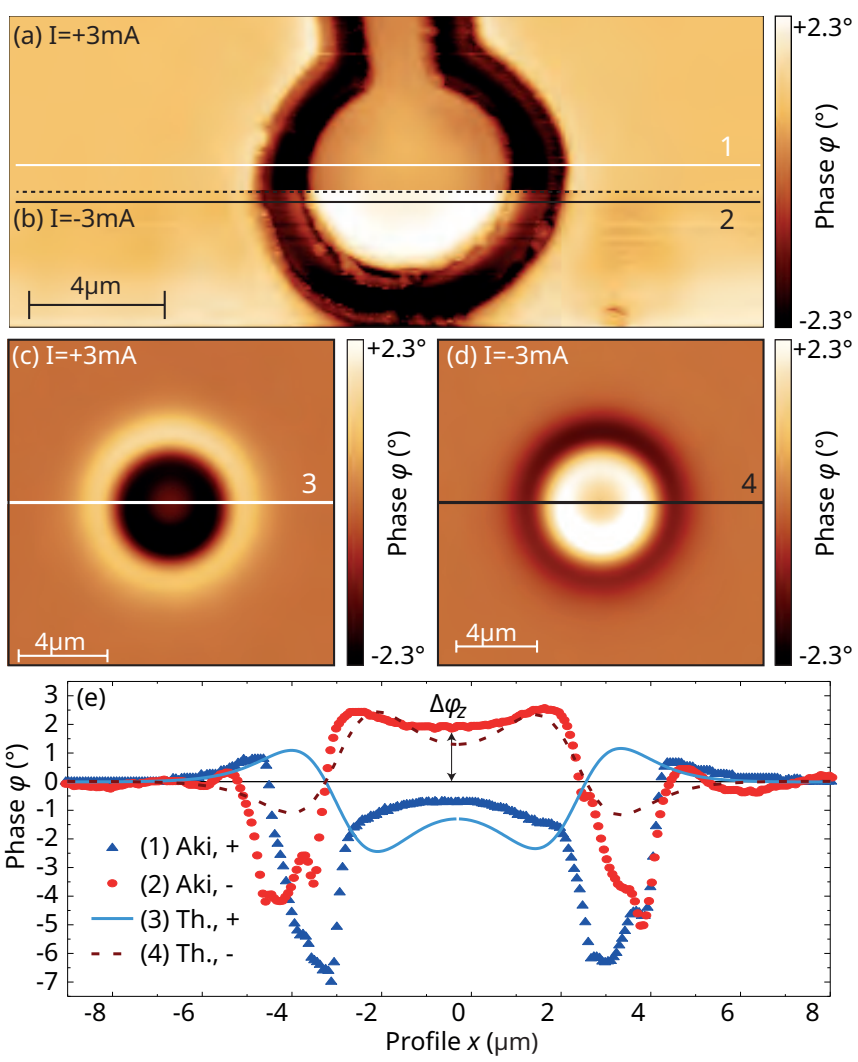

Fig. 4. MFM images of a current loop using an Akiyama tip at $T=4.2 \mathrm{~K}$, with applied currents of $I=+3 \mathrm{~mA}$ (a) and $I=-3 \mathrm{~mA}$ (b) and a scan height of $d=100 \mathrm{~nm}$. ((c) and (d)) show the corresponding simulations and (e) shows the four spectra as indicated.

$B_{c} \approx \pm 314 \mu \mathrm{T}$. The phase shift for positive applied current is $\Delta \varphi_{z,+}=-0.22^{\circ}$, and for a negative current, $\Delta \varphi_{z,-}=-0.14^{\circ}$, respectively. This shows that the electrostatic interaction play an important role when using Akiyama tips for MFM, when the tip and the cantilever are electrically connected to the tuning fork. This becomes even more evident when taking the MFM images into account, where large parts of the loop show black dots, where the tip has struck the gold surface. The phase at the center is also influenced by this effect, because the Akiyama tip is tilted, as can be seen in Fig. 1(b), and thus, even when the magnetic tip is in the center of the loop, part of the tip is above the current carrying Au wire. Topographic effects can be ruled out as source of the negative phase shift, since there is no significant signal when measuring with no applied current, see Figs. 3(g) and (h).

A more detailed characterization was possible at $T=4.2 \mathrm{~K}$. The MFM images of a current loop can be seen in Fig. 4(a) and (b), for applied currents of $I= \pm 3 \mathrm{~mA}$, the field at the center of the loop is $B_{c} \approx 783 \mu \mathrm{T}$, at a scan height of $d=100 \mathrm{~nm}$. Figs. 4(c) and (d) show the corresponding simulations using Eq. (4), the parameters $m_{z}$ and $\delta$ are obtained 
through fit of $\Delta \varphi_{z}(d)$ and $\Delta \varphi_{z}(I)$ to Eq. (5), as is explained below. The four spectra, as indicated in Figs. 4((a)-(d)), are shown in Fig. 4(e). The phase shift for positive applied current is $\Delta \varphi_{z,+}=-0.7^{\circ}$, and for a negative current, $\Delta \varphi_{z,-}=1.9^{\circ}$. The simulations are shown as (dotted) lines. For both applied currents, experiment and theory agree well. Deviations are obvious when measuring across the $\mathrm{Au}$ wire. This is due to the width of the wire of $\approx 1 \mu \mathrm{m}$, which is not taken into account in the simulations (a perfect current loop with zero width). Furthermore, the tip has a finite size and is not a perfect magnetic dipole. Both cause a broadening of the signal compared to theory. Also, a large negative contribution to the phase is evident when scanning above the gold. This is due to the electrostatic interaction between the current carrying loop and the Akiyama tip, when the tip hits the surface of the gold wire. The measurement of zero applied current did not show any significant signal (not shown here), therefore topographic effects can be ruled out as source of the negative phase. The phase shifts at the center of the loop show a larger value for both currents, when compared to the predicted one, see Fig. 4(e). This indicates an additional repulsive force $\left(\Delta \varphi>0\right.$ and $\left.F^{\prime}<0\right)$. The sum $\Delta \varphi_{z, \pm}=\left|\Delta \varphi_{z,+}\right|+\left|\Delta \varphi_{z,+}\right|=2.6^{\circ}$. This agrees with the simulations, which predicts $\Delta \varphi_{z, \pm}^{t h}=2.6^{\circ}$. Further, based on Figs. 4(a) and (b), an angle of the tip magnetization was taken into account, i.e. $\theta \approx 17^{\circ}$ and $\phi \approx 81^{\circ}$, as defined in Fig. 2. This yields a total tip magnetic moment of $\left|m^{*}\right|=2.42 \times 10^{-11} \mathrm{Am}^{2}$.

Using Eq. (5), we can get information about the effective magnetic moment of the tip in $z$-direction, $m_{z}$, and the dipole distance from the tip peak, $\delta$. For this purpose, the $y$-scan axis was fixed at zero and the center line was scanned several times. The resulting spectra were averaged, in order to minimize the noise. First, the phase shift at the center of the loop was measured as function of applied current, see Fig. 5(a). The positive offset was corrected. As is expected from Eq. (5), $\Delta \varphi(I)$ is linear, the straight red line is the fit. The current dependence can also be seen in the MFM image in Fig. 5(b). There, the $y$-scan axis was fixed at $y=0$ and the current was sweeped from $I=+5 \mathrm{~mA}$ to $I=-5 \mathrm{~mA}$. Similarly, the phase shift can be measured as a function of scan height as is shown in Fig. 6. The red line is the fit to Eq. (5). From the fits of the current and height dependence, an effective magnetic moment for the tip in $z$-direction $m_{z}=(2.4 \pm 0.5) \times 10^{-11} \mathrm{Am}^{2}$ and dipole distance $\delta=(1.85 \pm 0.1) \mu \mathrm{m}$ were obtained for the current loop with radius $a=2.4 \mu \mathrm{m}$. These values are of the same order of magnitude as those of other self made MFM tips [23, 24], and several orders of magnitude larger than the effective magnetic moment of commercial tips, where $\mathrm{a} \approx 50 \mathrm{~nm}$ layer of $\mathrm{CoCr}$ is usually used.

In order to obtain the coercive field of the magnetic Akiyama tip, a magnetic field of $7 \mathrm{~T}$ was applied prior to the measurements, in order to magnetize the tip in $z$-direction. The field was turned off, and as before, the
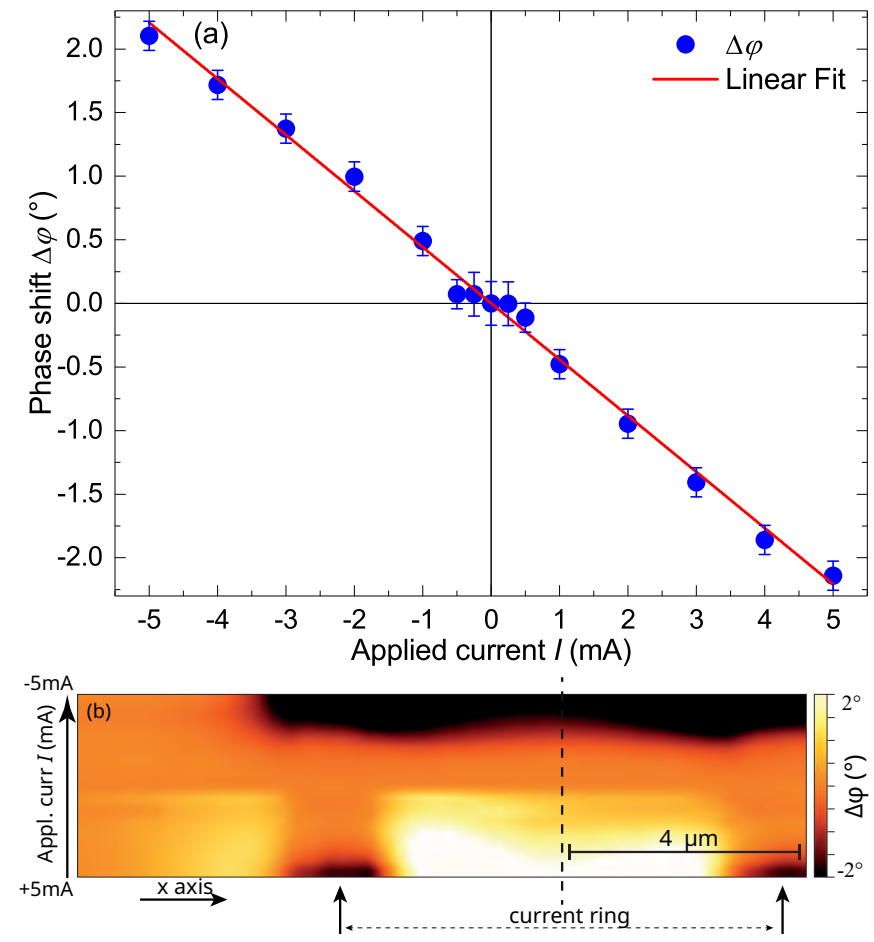

Fig. 5. (a) Phase shift $\Delta \varphi$ as function of applied current at $T=4.2 \mathrm{~K}$. The red line is the fit to Eq. (5). In (b) $\Delta \varphi$ vs. $I$ can be seen for a fixed $y$-scan axis.

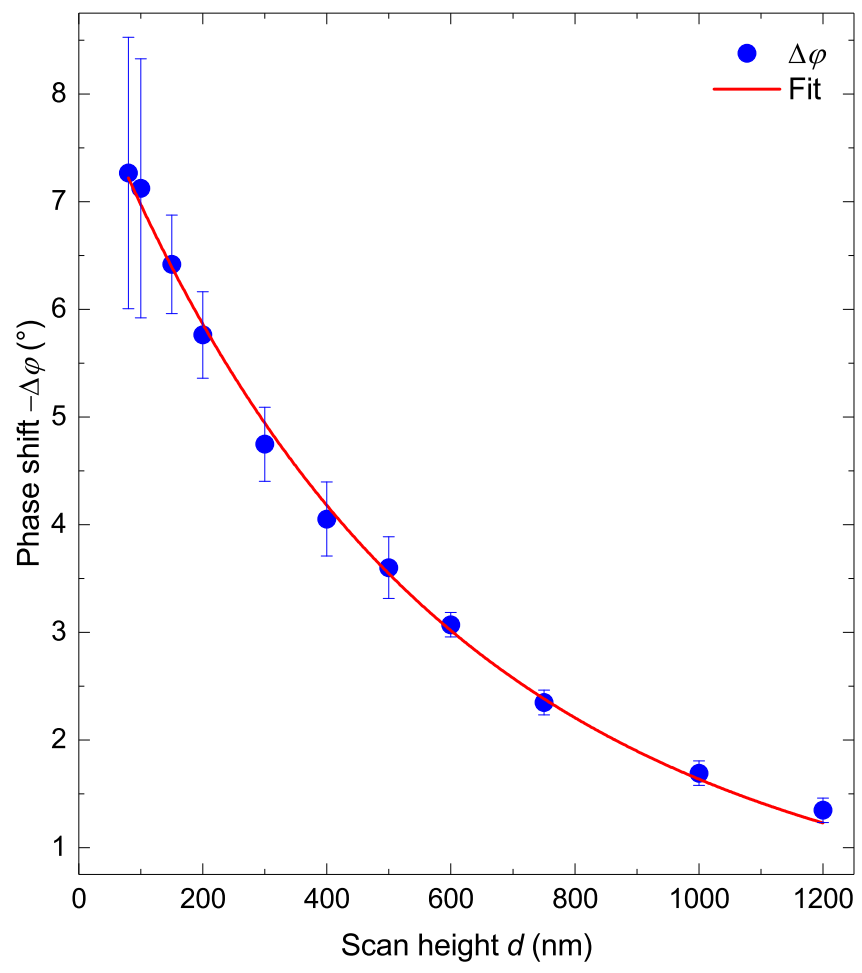

Fig. 6. Phase shift $\Delta \varphi$ as function of scan height $d$ at $T=4.2 \mathrm{~K}$. The red line is the fit to Eq. (5). 


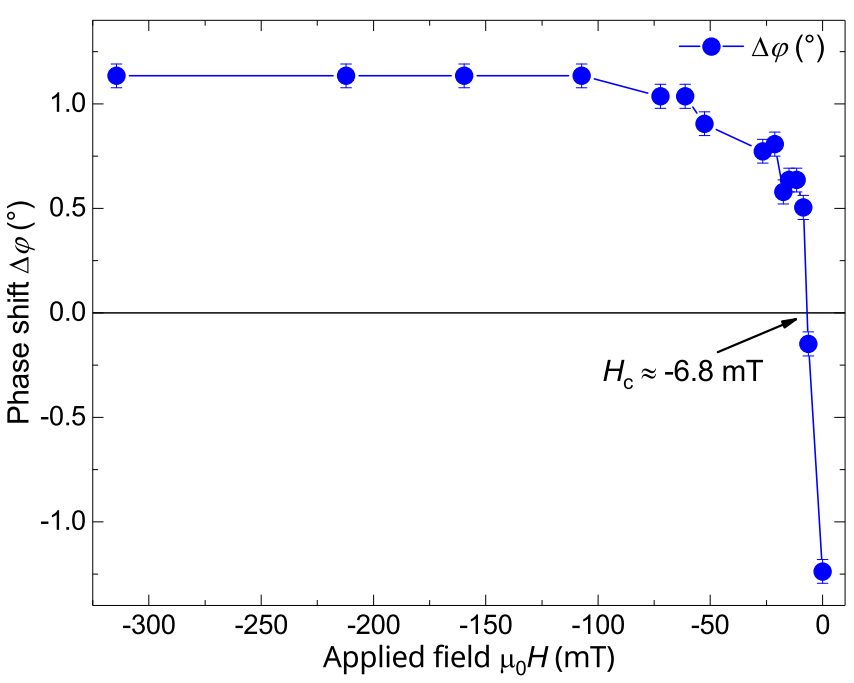

Fig. 7. Phase shift $\Delta \varphi$ as a function of magnetic field $H$ applied in the opposite direction of tip magnetization, at $T=4.2 \mathrm{~K}$.

$y$ axis was fixed at $y=0$ in order to scan the center line across the loop. Several scans have been measured and the phase signal was averaged. After each measurement the oscillation of the tip was turned off, and a magnetic field was applied in the opposite direction, and turned off again before measurement. The results can be seen in Fig. 7. A coercive field of $\approx 6.8 \mathrm{mT}$ was found and the magnetic saturation sets in at field larger than $0.1 \mathrm{~T}$.

\section{CONCLUSION}

FEBID of Co has been successfully used to produce magnetic Akiyama tips suitable for MFM measurements at $300 \mathrm{~K}$ as well as at low temperatures. Using FEBID, ferromagnetic Co can be deposited in sufficient amount on Akiyama tips and other standard AFM probes, in any desired shape and size. The results can be very well described using standard theories. A high spatial resolution is in principle feasible with the presented approach, given that narrow magnetic tips can be grown by FEBID [25]. Akiyama tips are sensitive to electrostatic interactions. Therefore, care has to be taken when measuring magnetic samples without an equipotential surface. Such self made magnetic tips can be used for MFM measurements with all the advantages of such self-oscillating and self-sensing tips, such as temperature dependent or lightsensitive measurements.

\section{ACKNOWLEDGMENTS}

This work was supported by the European cooperation in science and technology (COST) through COST Action CM1301, "Chemistry for Electron-Induced Nanofabrication" (CELINA), by the DFG through the Collaborative Research Center SFB 762 "Functionality of Oxide Interfaces" and by the DFG-ES86/29-1. This work was supported by Spanish Ministry of Economy and Competitivity through projects No. MAT2014-51982-C2-1-R and MAT2015-69725-REDT (including FEDER funds).
[1] J. Kirtley, M. Ketchen, K. Stawiasz, J. Sun, W. Gallagher, S. Blanton, and S. Wind, Appl. Phys. Lett. 66, 1138 (1995).

[2] A. Moser, H. Hug, B. Stiefel, and H.-J. Gúntherodt, J. of Magn. and Magn. Mat. 190, 114 (1998).

[3] A. Volodin, K. Temst, Y. Bruynseraede, C. Haesendonck, M. Montero, I. K. Schuller, B. Dam, J. Huijbregtse, and R. Griessen, Physica C: Superconductivity 369, 165 (2002).

[4] O. M. Auslaender, L. Luan, E. W. Straver, J. E. Hoffman, N. C. Koshnick, E. Zeldov, D. A. Bonn, R. Liang, W. N. Hardy, and K. A. Moler, Nature Physics 5, 35 (2008).

[5] M. Hehn, S. Padovani, K. Ounadjela, and J. Bucher, Phys. Rev. B 54, 3428 (1996).

[6] P. Schiffer, A. Ramirez, W. Bao, and S.-W. Cheong, Phys. Rev. Lett. 75, 3336 (1995).

[7] J. García, A. Thiaville, and J. Miltat, Appl. Phys. Lett. 79, 656 (2001).

[8] G. Chen, M. I. Bodnarchuk, M. V. Kovalenko, G. Springholz, W. Heiss, W. Jantsch, E. Platzgummer,
H. Loeschner, and J. Schotter, Adv. Mater. 22, 1364 (2010).

[9] L. Rondin, J.-P. Tetienne, P. Spinicelli, C. D. Savio, K. Karrai, G. Dantelle, A. Thiaville, S. Rohart, J.-F. Roch, and V. Jacques, Appl. Phys. Lett. 100, 153118 (2012).

[10] M. Serri, M. Mannini, L. Poggini, E. Vélez-Fort, B. Cortigiani, P. Sainctavit, D. Rovai, A. Caneschi, and R. Sessoli, Nano Letters 17, 1899 (2017).

[11] G. Lorusso, M. Jenkins, P. González-Monje, A. Arauzo, J. Sesé, D. Ruiz-Molina, O. Roubeau, and M. Evangelisti, Adv. Mater. 25, 2984 (2013).

[12] E. Pinilla-Cienfuegos, S. Mañas-Valero, A. FormentAliaga, and E. Coronado, ACS Nano 10, 1764 (2016).

[13] J. M. D. Teresa, A. Fernández-Pacheco, R. Córdoba, L. Serrano-Ramón, S. Sangiao, and M. R. Ibarra, J. Phys. D: Appl. Phys. 49, 243003 (2016).

[14] I. Utke, P. Hoffmann, R. Berger, and L. Scandella, Appl. Phys. Lett. 80, 4792 (2002). 
[15] H. Lavenant, V. Naletov, O. Klein, G. de Loubens, L. Casado, and J. M. de Teresa, Nanofabrication 1, 65 (2014).

[16] J. Pablo-Navarro, C. Magén, and J. M. de Teresa, Nanotechnology 27, 285302 (2016).

[17] G. Arnold, R. Timilsina, J. Fowlkes, A. Orthacker, G. Kothleitner, P. D. Rack, and H. Plank, ACS Appl. Mater. Interfaces 6, 7380 (2014).

[18] S. Sangiao, C. Magén, D. Mofakhami, G. de Loubens, and J. M. D. Teresa, accepted in Beilstein J. of Nanot.; ArXiv:1709.09374 xx, xx (xx).

[19] J. Simpson, J. Lane, C. Immer, and R. Youngquist, NASA Technical Report Server , 20010038494 (2001).

[20] W. Baumeister, P. Griitter, R. Guckenberger, H.-J. Güntherodt, T. Hartmann, H. Heinzelmann, H. Mamin,
E. Meyer, D. Pohl, D. Rugar, H. Siegenthaler, U. Staufer, H. Wickramasinghe, W. Wiegrabe, and R. Wiesendanger, Scanning Tunneling Microscopy II (Springer, 1992).

[21] A. Carl, J. Lohau, S. Kirsch, and E. Wassermann, J. of Appl. Phys. 89, 6098 (2001).

[22] K. Babcock, V. Elings, J. Shi, D. Awschalom, and M. Dugas, Appl. Phys. Lett. 69, 705 (1996).

[23] J. Lohau, S. Kirsch, A. Carl, G. Dumpich, and E. Wassermann, J. of. Appl. Phys. 86, 3410 (1999).

[24] L. Kong and S. Y. Chou, Appl. Phys. Lett. 70, 2043 (1997).

[25] J. Pablo-Navarro, D. Sanz-Hernández, C. Magén, A. Fernández-Pacheco, and J. M. de Teresa, J. Phys. D: Appl. Phys. 50, 18LT01 (2017). 